\title{
Testing split supersymmetry with inflation
}

\author{
Nathaniel Craig ${ }^{a}$ and Daniel Green ${ }^{b, c}$ \\ ${ }^{a}$ Department of Physics and Astronomy, Rutgers University, \\ 136 Frelinghuysen Rd, Piscataway, NJ 08854, U.S.A. \\ ${ }^{b}$ Stanford Institute for Theoretical Physics, Stanford University, \\ 382 Via Pueblo Mall, Stanford, CA 94305, U.S.A. \\ ${ }^{c}$ Kavli Institute for Particle Astrophysics and Cosmology, Stanford University, \\ 452 Lomita Mall, Stanford, CA 94305, U.S.A.
}

E-mail: ncraig@physics.rutgers.edu, drgreen@stanford.edu

ABSTRaCT: Split supersymmetry (SUSY) - in which SUSY is relevant to our universe but largely inaccessible at current accelerators - has become increasingly plausible given the absence of new physics at the LHC, the success of gauge coupling unification, and the observed Higgs mass. Indirect probes of split SUSY such as electric dipole moments (EDMs) and flavor violation offer hope for further evidence but are ultimately limited in their reach. Inflation offers an alternate window into SUSY through the direct production of superpartners during inflation. These particles are capable of leaving imprints in future cosmological probes of primordial non-gaussianity. Given the recent observations of BICEP2, the scale of inflation is likely high enough to probe the full range of split SUSY scenarios and therefore offers a unique advantage over low energy probes. The key observable for future experiments is equilateral non-gaussianity, which will be probed by both cosmic microwave background (CMB) and large scale structure (LSS) surveys. In the event of a detection, we forecast our ability to find evidence for superpartners through the scaling behavior in the squeezed limit of the bispectrum.

Keywords: Beyond Standard Model, Cosmology of Theories beyond the SM, Supersymmetric Standard Model

ArXiv EPRINT: 1403.7193 


\section{Contents}

1 Introduction 1

2 Split supersymmetry and its experimental probes 2

3 The reach of inflationary observables 5

4 Forecasts for an ideal experiment $\quad 8$

5 What can fake the signal? 13

6 Outlook 15

\section{Introduction}

The Standard Model has been completed by the discovery of an apparently elementary Higgs boson at the LHC. On one hand, the absence of evidence for additional degrees of freedom at the LHC challenges many proposals for new weak-scale physics beyond the Standard Model. On the other hand, the recent discovery of primordial tensor modes in the CMB by BICEP2 [1] points to the existence of new physics at a scale that suggestively coincides with apparent gauge coupling unification in supersymmetric extensions of the Standard Model [2]. That the scale indicated by cosmological observations coincides with the scale indicated by low-energy observations is extremely suggestive. In this paper we pursue the idea that cosmology may provide even more concrete evidence for the existence of supersymmetry (SUSY) well above the weak scale. ${ }^{1}$

Cosmological inflation [4-6] offers a novel opportunity to search for SUSY in the universe. The discovery of primordial tensor modes in the CMB by BICEP2 [1] strongly supports the idea that inflation occurred at very high energies. For the reported central value of $r=0.2_{-0.05}^{+0.07}$, the inflationary Hubble scale is given by $H \sim 1.1 \times 10^{14} \mathrm{GeV}$. Since any field with mass less than the inflationary Hubble scale can be produced during inflation, cosmological observables are sensitive to particles produced at these incredible energies.

Although the potential reach in energy of inflation is well-known, it has been less appreciated in the particle physics community that cosmological observables can directly test the presence of additional particles and interactions at these scales (see [7] for a recent review). One crucial observation is the single-field consistency condition $[8,9]$, which states that if inflation is described by a single degree of freedom then the bispectrum of the scalar curvature perturbation, $\zeta$, satisfies

$$
\lim _{\mathbf{k}_{3} \rightarrow 0}\left\langle\zeta_{\mathbf{k}_{1}} \zeta_{\mathbf{k}_{2}} \zeta_{\mathbf{k}_{3}}\right\rangle^{\prime} \rightarrow P_{\zeta}\left(k_{1}\right) P_{\zeta}\left(k_{3}\right)\left[\left(n_{s}-1\right)+\mathcal{O}\left(k_{3}^{2}\right)\right]
$$

\footnotetext{
${ }^{1}$ In some string models, having $m_{\frac{3}{2}}<H$ causes problems for moduli stabilization [3], which some authors take as evidence against low scale SƯ⿱
} 
Deviations from the consistency condition offer a relatively clean method for detecting additional fields present during inflation. The most commonly studied deviation is the case of local non-Gaussanity, where $\left(n_{s}-1\right) \rightarrow f_{\mathrm{NL}}^{\text {local }}$, which is most easily produced by additional massless scalars. On the other hand, massive scalars with $0<m \leq \frac{3}{2} H$ give rise to a bispectrum with soft limit [10]

$$
\lim _{\mathbf{k}_{3} \rightarrow 0}\left\langle\zeta_{\mathbf{k}_{1}} \zeta_{\mathbf{k}_{2}} \zeta_{\mathbf{k}_{3}}\right\rangle^{\prime} \rightarrow P_{\zeta}\left(k_{1}\right) P_{\zeta}\left(k_{3}\right)\left[\left(n_{s}-1\right)+\mathcal{O}\left(k_{3}^{\alpha}\right)\right]
$$

where $\alpha \equiv \frac{3}{2}-\sqrt{\frac{9}{4}-\frac{m^{2}}{H^{2}}}$. Measuring $\alpha<2$ both tells us that there is an extra degree of freedom and indicates its mass ${ }^{2}$ during inflation.

The above phenomenon provides a novel search technique for supersymmetry at high scales [13]. Although the non-observation of superpartners at the LHC is beginning to challenge scenarios of weak-scale supersymmetry, there remains strong motivation for socalled split supersymmetry scenarios where most or all superpartners lie outside the reach of the LHC [14-19]. If this is the course Nature has chosen, verifying the existence of supersymmetry at high scales requires new experimental probes. Provided that more direct sources of SUSY breaking scale are below the inflationary Hubble parameter, then the dominant source of SUSY breaking during inflation is set by the curvature, namely $H$. As a result, we expect to find additional scalar particles with masses set by $H$ that naturally produce signatures at a detectable level [13, 20]. A detection of $\alpha \sim 1$ would then provide tantalizing evidence that SUSY is relevant to our universe, even if it is never probed directly at the LHC (see also [21] for a different approach).

In this paper, we will explore the capability of cosmological observables to shed light on SUSY at high scales. In section 2 we review the low-energy evidence in support of supersymmetry at high scales, including the success of precision gauge coupling unification and the observed Higgs mass. In particular, the observed Higgs mass provides a suggestive upper bound on the present scale of SUSY breaking. In section 3, we then discuss the reach of cosmological observations in terms of the scale of SUSY breaking. We will discuss the assumptions that go into the predicted signals and how these compare with existing indirect probes. In section 4 , we will forecast our ability to detect $0<\alpha<2$ in an ideal $3 \mathrm{~d}$ experiment, with an eye towards large scale structure surveys. In section 5, we present possible alternative explanations of such a signal and how one could try to distinguish them. We conclude in section 6 with a discussion of the prospects for observation. Although the BICEP2 measurement of primordial tensor modes provides strong motivation, our study remains relevant irrespective of future changes in the central value of $r$.

\section{Split supersymmetry and its experimental probes}

The apparent unification of Standard Model gauge couplings under extrapolation to higher energies has long been a suggestive indication of new physics many orders of magnitude

\footnotetext{
${ }^{2}$ Strictly speaking, weakly coupled massive particles only produce $\alpha \leq \frac{3}{2}$. Taking $m>\frac{3}{2} H$ does not extend this limit, as these massive fields can be integrated out, up to exponentially suppressed contributions [11]. There is no obstacle to producing the full range $0 \leq \alpha<2$ with additional fields, as was demonstrated concretely in [12].
} 
above the weak scale [2]. Although gauge coupling unification in the context of the Standard Model alone is badly disfavored by both precision measurements from LEP and SLC and by the non-observation of proton decay, it is highly successful in supersymmetric extensions of the Standard Model. The additional matter content dictated by supersymmetry - particularly electroweak doublet fermions - places the supersymmetric prediction for gauge coupling unification in reasonable agreement with precision data and lowers the rate for proton decay consistent with current limits. It also picks out a particular scale for gauge coupling unification, $M_{\mathrm{GUT}} \sim 2 \times 10^{16} \mathrm{GeV}$, which is suggestively close to the scale of the inflationary potential favored by the observation of primordial tensor modes. In the conventional paradigm of weak-scale supersymmetry, supersymmetric unification can align within $\sim 3 \sigma$ of current low-energy data and may be reconciled with a modest $3-4 \%$ threshold correction at the unification scale [22]. However, the non-observation of superpartners at the LHC is beginning to put this paradigm under stress.

Surprisingly, the success of supersymmetric gauge coupling unification is improved in split supersymmetry [14-16], a scenario where scalar superparters lie well above the weak scale while fermionic superpartners are further protected by an $R$-symmetry and remain light. In this scenario supersymmetry no longer accounts for the entirety of the hierarchy between the weak scale and the Planck scale, although it still protects the weak scale against radiative corrections over many decades in energy. As the scalars are made heavy, the supersymmetric prediction for gauge coupling unification aligns perfectly with low-energy data without relying upon additional threshold corrections [14-16]. Fermionic superpartners are favored to remain light, since the contributions to running couplings from the light superpartners of the Higgs boson, the higgsinos, are largely responsible for successful unification. The precision of the unification prediction is illustrated in figure 1, which makes apparent that unification prefers light fermionic superpartners, although the preferred mass range for both scalars and fermions depends on the details of the fermionic spectrum. The inferred unification scale depends primarily on the higgsino mass, varying weakly from $M_{\mathrm{GUT}} \sim 5 \times 10^{15}-2 \times 10^{16} \mathrm{GeV}$ as the higgsinos vary from $\mu \sim 10^{2}-10^{4} \mathrm{GeV}$.

The case for some form of split supersymmetry is bolstered by the observation of a Standard Model-like Higgs of mass $m_{h} \sim 126 \mathrm{GeV}$. In minimal supersymmetric extensions of the Standard Model, the Higgs quartic is fixed by supersymmetry and radiative corrections due to supersymmetry breaking. In the case of split supersymmetry with light gauginos and heavy scalars, the observed Higgs mass is consistent with scalar superpartners in the range $\tilde{m} \sim 10^{4}-10^{8} \mathrm{GeV}[23]$. This favors the scenario of "mini-split" supersymmetry [17-19], in which scalars lie within six orders of magnitude of the weak scale - a subset of the possible range available in the original incarnation of split supersymmetry.

The bound on scalar superpartners in mini-split supersymmetry is fairly robust. Extensions of the MSSM that introduce additional quartic couplings typically lower the upper bound on $\tilde{m}$ by increasing the tree-level prediction for the Higgs mass. It is possible to raise the bound on $\tilde{m}$ if $A$-terms are large enough to induce negative threshold corrections to the quartic coupling, but this typically leads to prohibitive charge- and color-breaking minima well before the mass bound is substantially weakened. Alternately, if both scalar and gaugino masses are well above the weak scale (so-called "heavy supersymmetry", dis- 

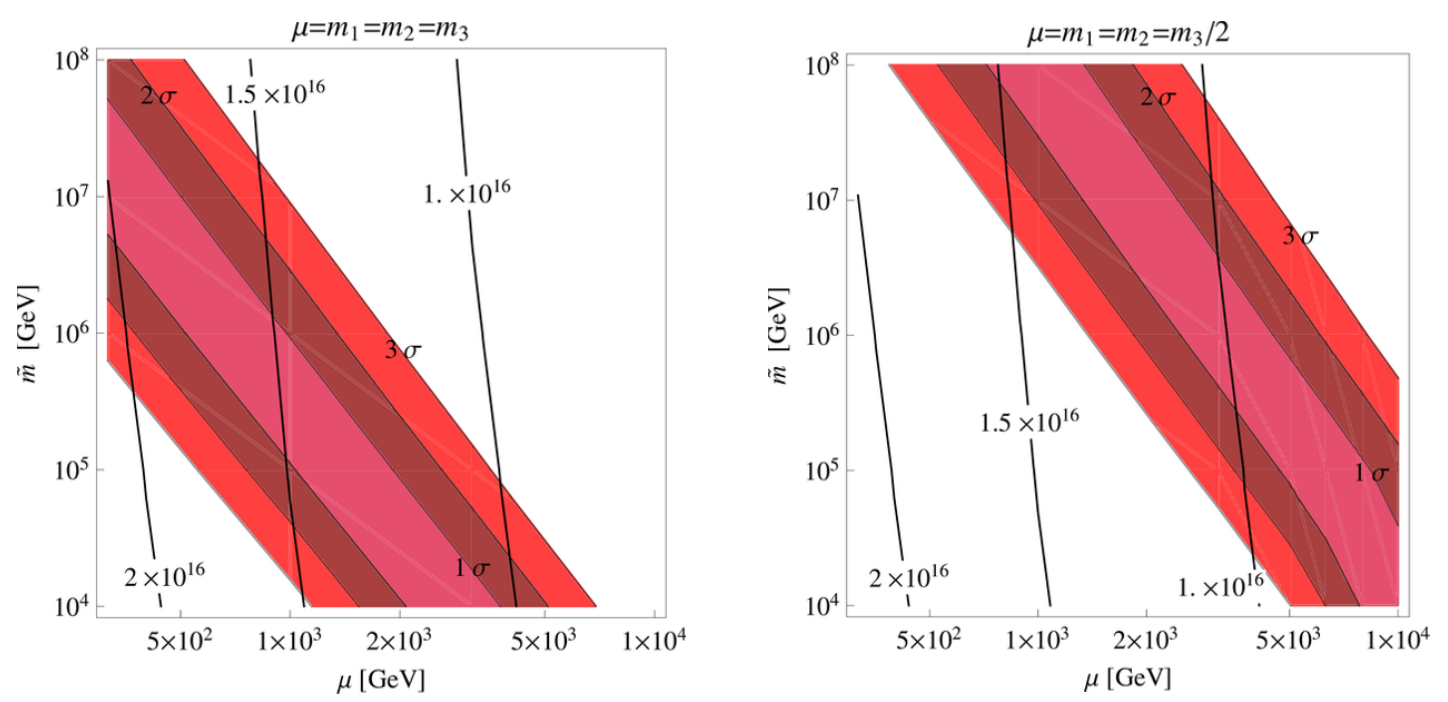

Figure 1. Unification prediction in split supersymmetry as a function of common fermionic $(\mu)$ and scalar $(\tilde{m})$ superpartner masses using two-loop running [14-16], neglecting weak-scale and unification-scale thresholds. In the left panel we take a common physical mass scale for all fermionic superpartners, while in the right panel we take the gluino to be twice as heavy as the other fermions. The diagonal bands represent the $1 \sigma, 2 \sigma, 3 \sigma$ constraints corresponding to $\alpha_{3}\left(M_{Z}\right)=0.1184 \pm 0.0007$, where we have taken the experimental inputs $\alpha_{e m}^{-1}\left(M_{Z}\right)=127.916$ and $\sin ^{2} \theta_{W}\left(M_{Z}\right)=0.23116$. The solid black contours indicate the one-loop unification scale in units of $\mathrm{GeV}$.

tinct from split supersymmetry), the running of the Higgs quartic changes such that the mass bound is relaxed to $\tilde{m} \lesssim 10^{13} \mathrm{GeV}$ [23], but at the cost of sacrificing precision gauge coupling unification if fermionic superpartners are heavier than $\sim 10^{6} \mathrm{GeV}$ [17-19].

In the framework of split supersymmetry, the mass bound on scalars can be translated into an upper bound on the scale of supersymmetry breaking in the present vacuum. There are generic Planck-scale contributions to scalar masses of order $\delta \tilde{m}_{\text {grav }} \sim F /\left(\sqrt{3} M_{\mathrm{pl}}\right)$, which implies $\sqrt{F} \lesssim 2 \times 10^{13} \mathrm{GeV}$. Low-scale mediation mechanisms such as gauge mediation entail even smaller values of $\sqrt{F}$. Although these contributions may be sequestered away, there remain anomaly-mediated contributions of order $\delta \tilde{m}_{\text {amsb }} \sim 10^{-2} \delta \tilde{m}_{\text {grav }}$, which implies at the very least $\sqrt{F} \lesssim 2 \times 10^{14} \mathrm{GeV}$. Thus the observed Higgs mass indicates that the scale of supersymmetry breaking in the present vacuum is, at its largest, two orders of magnitude below the scale of gauge coupling unification, assuming the boundary conditions for the Higgs quartic are set by split supersymmetry.

Finally, split supersymmetry may provide a viable dark matter candidate if neutral gauge fermions are sufficiently light and $R$-parity is conserved, though the dark matter candidate is subject to constraints from direct [24] and indirect [25, 26] searches. This is particularly attractive if the QCD axion with GUT-scale axion decay constant is no longer an effortlessly viable candidate for the majority of dark matter, as suggested by the combination of primordial tensor modes and isocurvature constraints [27].

While these indications are suggestive of split supersymmetry, they are not decisive. Precision unification, dark matter, and a viable Higgs mass prediction can all be achieved in non-supersymmetric extensions of the Standard Model, particularly if electroweak natural- 
ness is no longer a strong guide. Split supersymmetry may be probed directly if fermionic superpartners such as the gluino are within kinematic reach of the LHC, but this is far from guaranteed; gluinos may be kinematically inaccessible at the LHC without imperiling precision gauge coupling unification. Thus in order to determine whether supersymmetry is present at higher energies, indirect probes take on crucial significance.

At present the best indirect probes of split supersymmetry are precision observables such as electric dipole moments (EDMs) and flavor violation. EDMs are sensitive to new sources of $\mathrm{CP}$ violation in superpartner interactions, while flavor observables are sensitive to misalignment between fermion and scalar mass eigenstates and gain further sensitivity in the presence of additional $\mathrm{CP}$ violation.

There are two possible sources of EDMs in split supersymmetry. If the scalars are sufficiently light, there are one-loop diagrams contributing to EDMs involving loops of both scalar and fermionic superpartners, with $\mathrm{CP}$ violation arising through relative phases in SUSY-breaking soft parameters. If the new CP-violating phase is $\mathcal{O}(1)$, then the current electron EDM limit of $\left|d_{e}\right|<8.7 \times 10^{-29}$ [28] may be sensitive to scalars as heavy as $\tilde{m} \sim$ $2 \times 10^{5} \mathrm{GeV}$; the mass reach from limits on chromo-electric dipole moments is comparable. ${ }^{3}$ Alternately, there may be contributions to EDMs from two-loop diagrams that only involve fermionic superpartners, which dominate the EDM signal if scalars are sufficiently heavy. Current EDM limits may be sensitive to fermionic superpartners as heavy as $10^{3} \mathrm{GeV}$. In both cases, this is the maximum expected mass reach, assuming maximal phases and radiative contributions. Thus split supersymmetry does not guarantee an EDM signal, since the mass range of scalars and fermions may lie beyond the sensitivity of EDM experiments, or the size of new CP violation may be too small. On the other hand, the observation of an anomalous EDM would place a suggestive upper bound on the mass scale of split supersymmetry. ${ }^{4}$

The prospects for flavor violation are comparable to EDMs. The most sensitive observables involve $\mathrm{CP}$ violation in the kaon sector, where the maximal reach is currently $\tilde{m} \sim 10^{6} \mathrm{GeV}$ assuming maximal $\mathrm{CP}$ and flavor violation in the down-type squark sector [31]. These bounds are unlikely to improve significantly in the near future, though limits on flavor violation in other meson sectors may eventually achieve comparable sensitivity. The strength of indirect probes such as EDMs and flavor violation is that observation of an anomalous signal would place an upper bound on the mass scale of split supersymmetry on the order of $\tilde{m} \lesssim 10^{6} \mathrm{GeV}$. This also highlights the primary weakness: there is a vast range of scales in split supersymmetry consistent with gauge coupling unification and the observed Higgs mass that lie beyond the reach of these precision observables.

\section{The reach of inflationary observables}

The Hubble scale during inflation, $H$, is the characteristic energy at which fields are excited from the vacuum. Fields with mass $\lesssim H$ during inflation can contribute significantly to

\footnotetext{
${ }^{3}$ Here we allow large flavor violation in the scalar sector to maximize the possible reach in $\tilde{m}$ [29]; without large flavor violation the mass reach is an order of magnitude smaller [30].

${ }^{4}$ Though even in this case, there is no guarantee that an anomalous EDM is an indication of supersymmetry; an anomalous EDM could be spoofed by various new degrees of freedom such as a CP-violating extended Higgs sector.
} 
cosmological observables at later times. If supersymmetry is relevant to inflation, then it is necessarily broken by the curvature of space-time, which is also set by $H$. In this case we expect the masses of superpartners to be on the order of the Hubble scale during inflation but not parametrically larger. Concretely, in old minimal supergravity, the scalar partner of the inflaton receives a universal contribution of $m=2 H$ [32] from its curvature coupling alone, which is modified by model dependent contributions from gravity mediation. As a result, superpartners will be produced from the vacuum for any value of $H$, provided that $H$ is the largest source of SUSY breaking. This structure is further motivated by attempts to make inflation technically natural [13], although the large field range implied by the tensor amplitude $[33,34]$ introduces additional challenges for producing a viable model of inflation.

As we have seen, in the context of split supersymmetry the scale of SUSY breaking in the current vacuum is at most $\sqrt{F} \sim 10^{13-14} \mathrm{GeV}$ and in general can be significantly smaller. For this reason, it is very plausible that supersymmetry is relevant to inflation, particularly if the Hubble scale during inflation is on the order of $H \sim 10^{14} \mathrm{GeV}$ as suggested by BICEP2. Thus we expect to leverage the full power of supersymmetric signatures of inflation to probe scenarios of split supersymmetry.

Given these general considerations, the presence of an extra scalar field $\sigma$ with a mass $m \sim H$ is a very natural consequence of SUSY in our universe. However, in order to produce measurable deviations from the single-field consistency conditions, it must also couple to the inflaton, $\phi$, as was first studied in [10] under the name quasi-single field inflation (QSFI). For our purposes it suffices to take the full Lagrangian for such a scalar field $\sigma$ to be

$$
\mathcal{L}_{\sigma}=-\frac{1}{2}\left[\partial_{\mu} \sigma \partial^{\mu} \sigma+m^{2} \sigma^{2}\right]-\mu \sigma^{3}+\frac{\sigma}{\Lambda}\left[\partial_{\mu} \phi \partial^{\mu} \phi-\langle\dot{\phi}\rangle^{2}\right]
$$

where we expect $m \sim H$ and otherwise remain agnostic about the size of $\mu$ and $\Lambda$. We have coupled $\sigma$ to $\phi$ derivatively in order to protect the approximate scale invariance of the observed power spectrum (which is enforced by an approximate shift symmetry, $\phi \rightarrow \phi+c)$. During inflation, $\dot{\phi}$ acquires a vev that introduces a tadpole for $\sigma$, which we have cancelled explicitly ${ }^{5}$ since we wish to study fluctuations around the minimum of the potential. Fluctuations in $\sigma$ are converted into fluctuations in $\phi$ through the $\Lambda$ suppressed coupling. Self-interactions of $\sigma$ therefore constitute the leading contribution to the bispectrum, with the shape of the non-gaussianity interpolating between local ( $m \ll H$ ) and equilateral $(m \sim H)$. Furthermore, the squeezed limit of the bispectrum bears the imprint of the nonzero $\sigma$ mass.

It is important to note that even Planck-suppressed interactions (i.e., $\Lambda \sim M_{\mathrm{pl}}$ ) are sufficient to generate a measurable signal $[12,20]$. The requisite couplings were studied carefully in [20], where for weak mixing $\left(\frac{\dot{\phi}}{\Lambda} \ll H\right)$ it was found that

$$
\frac{f_{\mathrm{NL}}^{\text {equil. }}}{75} \sim 12 \frac{\mu}{H}\left(\frac{r}{0.2}\right)^{1 / 2}\left(\frac{M_{\mathrm{pl}}}{\Lambda}\right)^{3} .
$$

\footnotetext{
${ }^{5}$ In general, $\dot{\phi}$ is time dependent and therefore the above formula is not correct as written. It is straightforward to enforce tadpole cancelation at all times by embedding this model in the effective field theory of inflation $[13,35,36]$. See [20] for further discussion.
} 
The existing constraint on $f_{\mathrm{NL}}^{\text {equil. }}$ from Planck is given by $f_{\mathrm{NL}}^{\text {equil. }}=-42 \pm 75$ (at $1 \sigma$ ), so we are already capable of measuring Planck-suppressed couplings for sufficiently large $\mu$, as was emphasized in [20]. Large-scale structure surveys are expected to improve on these measurements through the galaxy bispectrum with potential sensitivity of $\Delta f_{\mathrm{NL}}^{\text {equil. }} \sim 10$ [37] (or optimistically $\Delta f_{\mathrm{NL}}^{\text {equil. }}<1[38]$ ).

The natural question is then whether we generically expect additional scalars in split supersymmetry to possess the interaction terms in (3.1). Two very plausible, technically natural scenarios in which the above action can be generated are:

- if inflation is described by a single chiral superfield, $\sigma$ and $\phi$ can be the two real components of the complex scalar [13]. The mass $m \sim H$ for $\sigma$ is generated through gravity mediation and/or curvature couplings. The self-interaction of $\sigma$ and the mixing between $\sigma$ and $\phi$ both arise through shift-symmetric irrelevant operators in the Kähler potential. ${ }^{6}$ If these irrelevant operators are suppressed by powers of the same scale $\Lambda$ with comparable dimensionless coefficients, one typically expects $\Lambda \lesssim M_{\mathrm{pl}} / 10$ is necessary for an appreciable signal. However, it is also possible for $\mu \sim H$ accidentally (i.e. the irrelevant operator generating $\mu$ may have an anomalously large dimensionless coefficient), allowing an appreciable signal with $\Lambda \sim M_{\mathrm{pl}}$.

- When there are multiple light chiral superfields, $\sigma$ and $\phi$ can arise from different superfields. In this case the potential for $\sigma$ is less constrained; both $\mu$ and $m$ can be generated via gravity mediation [20] and are naturally of the correct size to produce a measurable signal with $\Lambda \sim M_{\mathrm{pl}}$.

We will be agnostic about which scenario is more plausible. In the first case, the degrees of freedom and self-interactions are intrinsic to supersymmetric inflation with irrelevant operators in the Kähler potential, but the generic scale of irrelevant operators required for a signal is somewhat below $M_{\mathrm{pl}}$. In the second case, the degrees of freedom and self-interactions are not intrinsic to supersymmetric inflation (but are highly plausible ingredients), while the scale of irrelevant operators can naturally be $\mathcal{O}\left(M_{\mathrm{pl}}\right)$. Both are well motivated from different model building perspectives and lead to potentially observable signatures.

\footnotetext{
${ }^{6}$ The three leading irrelevant operators of interest are $K \supset \frac{1}{\Lambda}\left(\Phi+\Phi^{\dagger}\right)^{3}, \frac{1}{\Lambda^{3}} X^{\dagger} X\left(\Phi+\Phi^{\dagger}\right)^{3}$, and $\frac{1}{\Lambda^{3}}\left(\Phi+\Phi^{\dagger}\right)^{5}$, where $\Phi$ is the superfield containing $\sigma, \phi$ while $X$ is a field seeding the value of the inflaton potential during inflation, $F_{X} \sim M_{\mathrm{pl}} H$ (see [13] for further discussion). The first operator gives rise to the mixing term and also generates a self-interaction of $\sigma$ via curvature couplings. The second and third operator give rise to self-interactions for $\sigma$ directly. The contributions to $\mu / H$ from these operators are of order $H / \Lambda, M_{\mathrm{pl}}^{2} H / \Lambda^{3}$, and $\mathcal{O}\left(10^{7}\right) H^{3} / \Lambda^{3}$, respectively, and all give rise to effects of similar numerical size. Note however that for the first operator, the same scale $\Lambda$ suppresses both the self-interaction and mixing terms; the naive $\Lambda$ required for an observable $f_{\mathrm{NL}}$ from this operator alone entails $\mathcal{O}(1)$ mixing. This lies outside the regime of validity of the weak mixing result (3.2) and a more detailed analysis of $f_{\mathrm{NL}}$ is required; see [20]. For the second and third operators, the $\Lambda$ can be slightly different from the scale suppressing mixing terms, generating an observable bispectrum while preserving the validity of the weak mixing result $(3.2)$.
} 
In either case, the signature of $m \sim H$ appears in the squeezed limit of the bispectrum. The behavior in the squeezed limit was worked out analytically in [10]:

$$
\lim _{k_{3} \rightarrow 0} B\left(k_{1}, k_{2}, k_{3}\right)=\frac{12}{5} f_{\mathrm{NL}}^{\text {equil. }} c_{\alpha} \times P_{\zeta}\left(k_{1}\right) P_{\zeta}\left(k_{3}\right)\left(\frac{k_{3}}{k_{1}}\right)^{\alpha}
$$

where $c_{\alpha} \sim \mathcal{O}(1)$. The observation of modest non-gaussianity and squeezed-limit scaling consistent with (3.3) would then be suggestive evidence for supersymmetry at high scales.

In practice, we can use the QSFI template [10] given by

$$
B^{\mathrm{QSFI}}\left(k_{1}, k_{2}, k_{3}\right)=\frac{18}{5} \Delta_{\zeta}^{4} f_{\mathrm{NL}}^{\text {equil. }} \frac{3^{3 / 2}}{N_{-\alpha+3 / 2}\left[\frac{8}{27}\right]} \frac{N_{-\alpha+3 / 2}\left[\frac{8 k_{1} k_{2} k_{3}}{\left(k_{1}+k_{2}+k_{3}\right)^{3}}\right]}{\left[k_{1} k_{2} k_{3}\left(k_{1}+k_{2}+k_{3}\right)\right]^{\frac{3}{2}}}
$$

where $N_{\nu}[x]$ is the Neumann function. It was shown in [39] that the above ansatz is in good agreement with the correct theoretical bispectrum in the equilateral, flattened and squeezed configurations away from $\alpha=0$ and is therefore sufficient ${ }^{7}$ for our purposes.

There is a strong analogy between the prospects for an inflationary signal of supersymmetry and low-energy probes such as EDMs and flavor violation. In the case of an inflationary signal, the degrees of freedom are intrinsic - at the very least, supersymmetry demands a real scalar partner of the inflaton and protects the mass of other light scalars. Although the signal is not guaranteed to be accessible by next-generation experiments, there is nonetheless a wide range of well-motivated scenarios where the signal is appreciable. Non-observation of equilateral non-gaussianity and/or a squeezed limit with $\alpha<2$ does not exclude supersymmetry, but observation of such a signal would provide a compelling indication for supersymmetry at high scales. In the case of low-energy probes the degrees of freedom are also intrinsic - supersymmetry demands superpartners of Standard Model fields - but the signal is also not guaranteed to be accessible; CP-violating phases or flavor violation might be too small to observe even if the mass scales are within reach. As such, non-observation of low-energy anomalies likewise does not exclude supersymmetry at high scales, while observation of a signal would be highly suggestive.

The distinct advantage of an inflationary signal over low-energy probes is that it remains sensitive to all scales of SUSY breaking allowed by split supersymmetry, and hence can probe SUSY breaking well beyond the reach of low-energy probes. That said, the finite reach of low-energy probes offers a complementary advantage: an anomalous EDM or FCNC could then be used to set a non-trivial upper bound on the scale of Standard Model superpartners.

\section{Forecasts for an ideal experiment}

The best limits on primordial non-gaussianity to date come from the CMB via the Planck satellite [40]. The bounds from the CMB could be further improved through a future

\footnotetext{
${ }^{7}$ In the limit $\alpha \rightarrow 0, \sigma$ becomes massless and the model becomes sensitive to physics at reheating. Although the QSFI template does not agree with the analytic calculations in this limit, both are missing potential late time contributions which would contribute to the local shape. Nevertheless, as our primary interest will be distinguishing $\alpha \sim 1$ from $\alpha=2$, we will not be concerned about inaccuracies around $\alpha=0$.
} 
polarization-sensitive satellite mission [41]. In addition, the coming generation of largescale structure (LSS) surveys can plausibly reach $\Delta f_{\mathrm{NL}}^{\text {equil. }} \sim 10$ [37] via measurements of the bispectrum of tracers of the LSS. In what follows we will focus on LSS as the parameters of the surveys are known, but similar considerations will apply to a future CMB experiment.

Our interest here is detecting deviations from the single field consistency condition (1.1) of the form (1.2). A clean detection of $\alpha<2$ unambiguously requires additional fields (beyond the inflaton) and for $\alpha>0$ suggestively points to SUSY. Although the specific model written in (3.1) makes predictions beyond the scaling in the squeezed limit, such as the detailed shape in equilateral configurations, these predictions are not robust to the inclusion of additional massive fields or self-interactions of the inflaton. For this reason, we are interested in isolating the the scaling behavior in the squeezed limit to determine $\alpha$.

Unfortunately, isolating the squeezed limit means our constraints will be weaker than if we used all the information at our disposal to constrain this specific model. Forecasts for the measurement of $\alpha$ using the full QSFI template were performed in [39, 42] and they indeed find stronger limits for a given $\bar{f}_{\mathrm{NL}}$. We will not follow the same strategy here to avoid using equilateral configurations to determine $\alpha$, as we wish to remain agnostic as to the underlying model of inflation. In addition, the confidence at which one can rule out $\alpha=2$ is the most unambiguous signal of SUSY, but the point $\alpha=2$ is not a welldefined value for the QSFI template. For this reason, [39, 42] cannot ${ }^{8}$ extend their forecasts beyond $\alpha=\frac{3}{2}$. Although both types of analyses would be important to perform on real data, we believe our more conservative analysis would be required to definitely rule out single field inflation.

For the purpose of understanding our reach in $\alpha$, we will consider an ideal $3 \mathrm{~d}$ measurement of the primordial correlation functions. For cosmic variance limited measurements in the linear regime, this should be a good approximation for the experimental sensitivity. Pushing $\Delta f_{\mathrm{NL}}^{\text {equil. }}<10$ will ultimately require modeling (mildly) non-linear structure formation and it is less clear that these estimates will translate directly. Nevertheless, these idealized estimates should provide a lower limit on the sensitivity of a real experiment.

Suppose we are given a fiducial model with some fiducial value of $\bar{f}_{\mathrm{NL}}$ and $\bar{\alpha}$. The likelihood function for $f_{\mathrm{NL}}$ and $\alpha$ for these fiducial values, assuming scale invariance, is given by $[43,44]$

$$
-2 \log \mathcal{L}=\frac{V}{\Delta_{\zeta}^{6}} \int \frac{d^{3} k_{1}}{(2 \pi)^{3}} \frac{1}{\left(4 \pi^{2}\right)} \int_{1 / 2}^{1-\epsilon_{*}} d x_{2} \int_{1-x_{2}}^{x_{2}} d x_{3}\left(B\left(1, x_{2}, x_{3}\right)-\bar{B}\left(1, x_{2}, x_{3}\right)\right)^{2} x_{2}^{4} x_{3}^{4}
$$

where $x_{2,3}=\frac{k_{2,3}}{k_{1}}, V=(2 \pi)^{3} / k_{\text {min }}^{3}$ is the spatial volume of the survey and $\bar{B}$ and $B$ are the bispectra with the fiducial and measured values of parameters, respectively. The parameter $\epsilon_{*}$ denotes the most squeezed configurations available in the survey, $\epsilon_{*} \equiv \frac{k_{\min }}{k_{\max }}$.

In this parameterization, the squeezed limit corresponds to $x_{2} \rightarrow 1$ and $x_{3} \rightarrow 0$. It is easy to see that for $\bar{\alpha}>0$, the dominant contribution to the likelihood function is not from the squeezed limit but from more equilateral configurations $\left(x_{2} \sim x_{3} \sim 1\right)$. In practice, this means this first signature of SUSY would be the detection of $f_{\mathrm{NL}}^{\text {equil. }}$. In addition, for

\footnotetext{
${ }^{8}$ The analysis of [42] also included a discussion of general scaling in the squeezed limit. This analysis differs from ours in several respects and is therefore not directly comparable.
} 


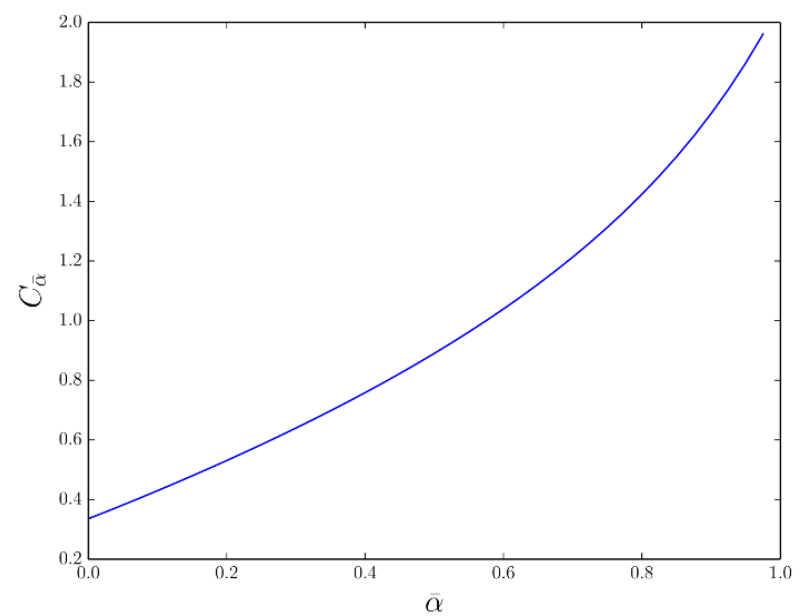

Figure 2. The relative normalization of the amplitude of the likelihood function in the squeezed and equilateral limits, as defined by $C_{\bar{\alpha}}$. The function was computed for varying fiducial values of $\bar{\alpha}$ using the QSFI template in (3.4).

$\alpha>0$ we are mildly sensitive to extremely squeezed configurations, which tend to differ significantly depending on the type of observation. As result, our forecasts should be fairly robust away from $\alpha=0$.

Our goal here is to understand the limits that we can place on $\alpha$ without reference to the equilateral configuration. If we isolate only the squeezed configurations with momenta in the range $\epsilon_{*}<x_{3}<\epsilon$, from our ansatz in (3.3), we have

$$
-2 \log \mathcal{L}=\frac{\left(f_{\mathrm{NL}}-\bar{f}_{\mathrm{NL}}\right)^{2}}{\sigma_{f_{\mathrm{NL}}}^{2}}+\frac{1}{\sigma_{f_{\mathrm{NL}}}^{2}} \int_{1-\epsilon}^{1-\epsilon_{\star}} \frac{d x_{2}}{x_{2}^{2}} \int_{1-x_{2}}^{\epsilon} \frac{d x_{3}}{x_{3}^{2}}\left[f_{\mathrm{NL}} C_{\alpha}\left(\frac{x_{3}}{x_{2}}\right)^{\alpha}-\bar{f}_{\mathrm{NL}} C_{\bar{\alpha}}\left(\frac{x_{3}}{x_{2}}\right)^{\bar{\alpha}}\right]^{2}
$$

where $\sigma_{\mathrm{f}_{\mathrm{NL}}}$ is the $1-\sigma$ error placed on $f_{\mathrm{NL}}^{\text {equil. }}$ from equilateral measurements and $C_{\alpha} \equiv$ $c_{\alpha} f_{\mathrm{NL}} / \sqrt{B \cdot B}$ with $B \cdot B \equiv \int_{1 / 2}^{1-\epsilon} d x_{2} \int_{\epsilon}^{x_{2}} d x_{3} B\left(1, x_{2}, x_{3}\right)^{2} x_{2}^{4} x_{3}^{4}$.

It is crucial that our determination of $\alpha$ comes only from the exponent in the squeezed limit. Therefore, to determine sensitivity to $\alpha$ we should marginalize over $f_{\mathrm{NL}}$ and $C_{\alpha}$. We will take a flat prior on $f_{\mathrm{NL}}$ for simplicity. This choice is reasonable because the likelihood function will be dominated by the leading term under the assumption that we detect the equilateral shape. We will also take a flat prior on $\tilde{C}_{\alpha} \equiv f_{\mathrm{NL}} C_{\alpha}$ in order to remain agnostic as to the relationship between $f_{\mathrm{NL}}^{\text {equil. }}$ and the amplitude in the squeezed limit. We will assume the fiducial $C_{\bar{\alpha}}$ is the one from QSFI, computed using the QSFI template (3.4) and shown as a function of $\bar{\alpha}$ in figure 2 .

The projected reach in $\alpha$ is shown in figures 3 and 4 for $\sigma_{f_{\mathrm{NL}}}=10$. We assume the squeezed limit applies for $\epsilon=10^{-1}$ and we take $\epsilon_{*}=\frac{k_{\min }}{k_{\max }} \sim 3 \times 10^{-3}$. The choice of $\epsilon_{*}$ is based on expectations from the Euclid survey [45] volume of $V=108 h^{-3} \mathrm{Gpc}^{3}$ and $k_{\max } \sim 0.4 \mathrm{~h} \mathrm{Mpc}^{-1}$ (which is fairly conservative for $1<z<2$ ). Taking advantage of scaledependent bias [46, 47] in the bispectrum may ultimately improve this effective range [48], but we will take this more conservative choice. Given that the signal is not concentrated in the squeezed limit, bias should not play a huge role beyond determining $\sigma_{f_{\mathrm{NL}}}$. 


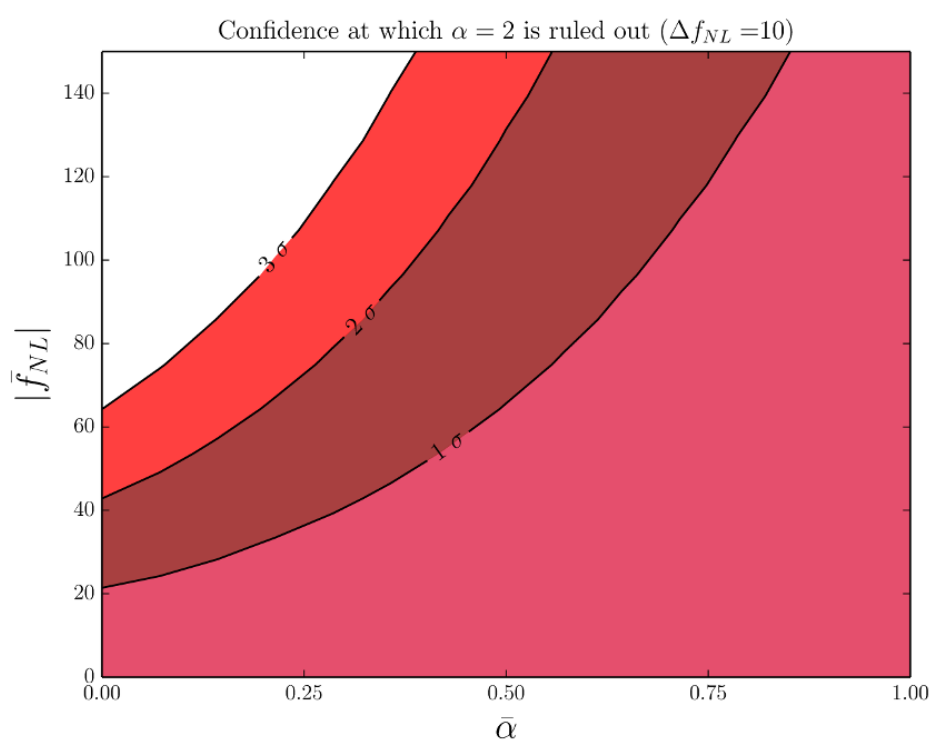

Figure 3. The confidence level at which $\alpha=2$ can be ruled out as a function of varying $\bar{f}_{\mathrm{NL}}$ and $\bar{\alpha}$. The forecasted contours use $\sigma_{f_{\mathrm{NL}}}=10, \epsilon=10^{-1}$ and $\epsilon_{*}=3 \times 10^{-3}$.

In figure 3, we address the key question of how well $\alpha=2$ can be excluded as a function of the fiducial values $\bar{f}_{\mathrm{NL}}$ and $\bar{\alpha}$. For values of $\bar{f}_{\mathrm{NL}}$ consistent with current Planck limits $\left|f_{\mathrm{NL}}\right|<117(1 \sigma), \alpha=2$ can be ruled out by as much as $3 \sigma$ for small values of $\bar{\alpha}$. Furthermore for $\left|f_{\mathrm{NL}}\right|>70$, discrimination is possible out to $\bar{\alpha}=0.25$ with more than $2 \sigma$, which corresponds to a scalar of mass

$$
m \lesssim 0.8 H
$$

validating the proposal that a scalar of mass $m \sim H$ can be probed by cosmological observables within the next generation of LSS and CMB polarization surveys. In figure 4, we then show the expected precision with which the borderline case of a fiducial value $\bar{\alpha}=0.25$ can be measured as a function of $\bar{f}_{\mathrm{NL}}$.

It bears emphasizing that our forecasts are relatively conservative. By ignoring equilateral configurations in measurements of $\alpha$, we are intentionally neglecting a lot of information. For example, our choice of $\epsilon \equiv\left(\frac{k_{1}}{k_{3}}\right)_{\min }$ defining the minimum required squeezing to be included in the measurement of $\alpha$ is somewhat arbitrary. In figure 5 we show how our ability to rule out $\alpha=2$ is sensitive to our choice of $\epsilon$. As we increase $\epsilon$, our ability to rule out $\alpha=2$ improves rapidly because the signal to noise is concentrated in the equilateral configurations. Even a modest change in $\epsilon$ substantially improves our discrimination.

Despite our somewhat conservative choice of $\epsilon=0.1$, the galaxy bispectrum of a single survey (e.g. Euclid or BOSS) is capable of a detection of equilateral non-gaussianity $(>5 \sigma)$

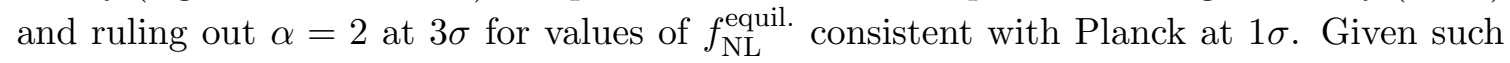
a detection, one could gain more significance for the detection of $\alpha<2$ through combined analysis with other probes (e.g. scale dependent bias, multiple tracers, CMB) and improved modeling of mildly nonlinear scales. 


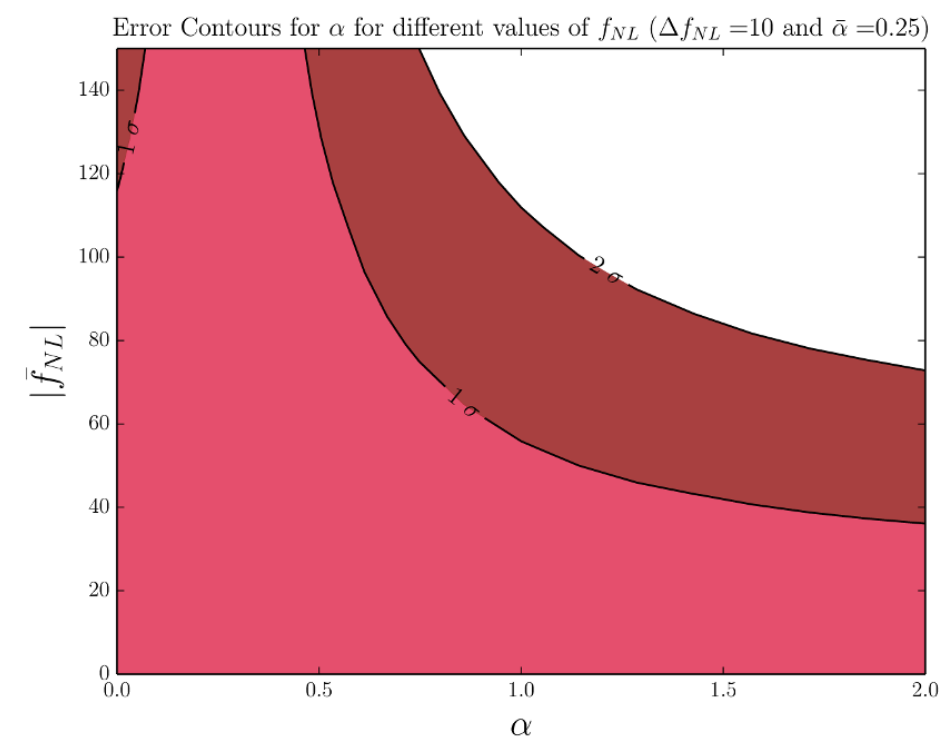

Figure 4. The $1 \sigma$ and $2 \sigma$ error contours on the measurement of $\bar{\alpha}=0.25$ for varying values of $\bar{f}_{\mathrm{NL}}$. The forecasted contours use $\sigma_{f_{\mathrm{NL}}}=10, \epsilon=10^{-1}$ and $\epsilon_{*}=3 \times 10^{-3}$. While the plot shows clear measurements of $\alpha<2$, it appears to show results compatible with $\alpha=0$. This is an artifact of our flat prior on $\tilde{C}$ and our assumption that the dominant signal is in the equilateral template. For $\bar{\alpha} \sim 0$, the squeezed limit dominates the signal to noise and we would see this as a detection of $f_{\mathrm{NL}}^{\text {local }}$.

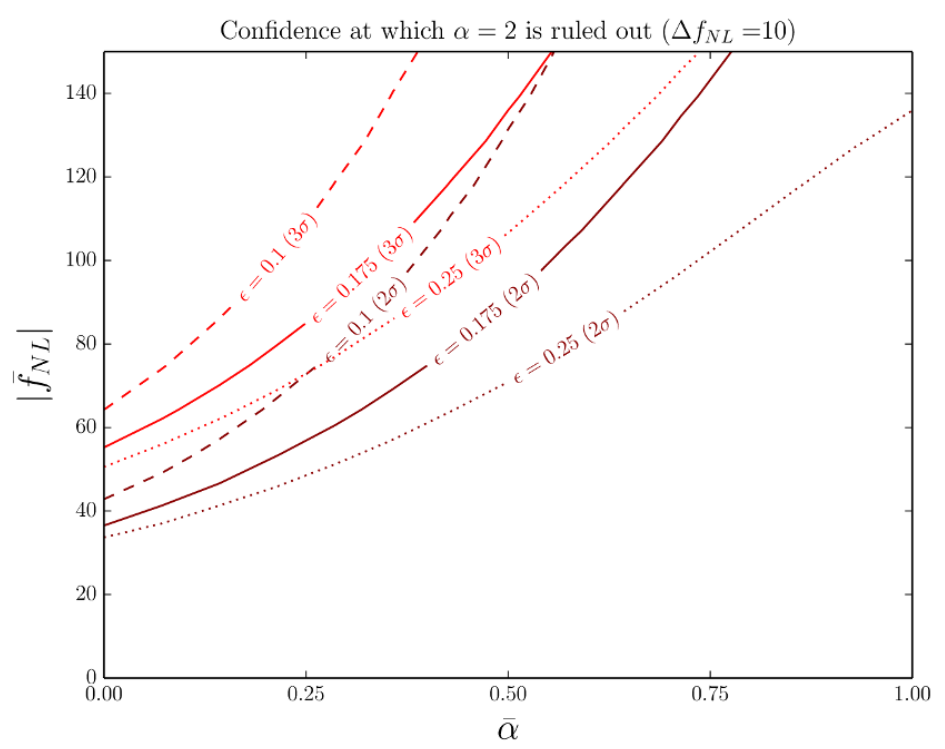

Figure 5. The confidence level at which $\alpha=2$ can be ruled out as a function of varying $\epsilon, \bar{f}_{\mathrm{NL}}$ and $\bar{\alpha}$. The forecasted contours use $\sigma_{f_{\mathrm{NL}}}=10$ and $\epsilon_{*}=3 \times 10^{-3}$. The $2 \sigma$ and $3 \sigma$ contours are shown for $\epsilon=0.1,0.175,0.25$. Our ability to rule out $\alpha=2$ improves significantly as we include more information from equilateral configurations. 


\section{What can fake the signal?}

Although the bispectrum is sensitive to SUSY during inflation, one might also wonder to what degree this is a unique signature. In other words, if we were to make a detection of $\alpha<2$, how confident would we be that there is supersymmetry in the universe?

Clearly, fully supersymmetrizing the Lagrangian in (3.1) entails adding fermions to complete the appropriate supermultiplets. While the fermions will also contribute to observables, their contributions are typically suppressed by a power of $\Delta_{\zeta}$. As a result, given current bounds on non-gaussianity, future surveys are not sufficiently sensitive to detect fermions with couplings related by SUSY. Rather, the crucial role of SUSY in our discussion was to explain the origin of a scalar with $m \sim H$ naturally. If one is simply willing to fine-tune a scalar to have the same Lagrangian and $m \sim H$ without radiative protection from a symmetry, then the dominant signal will be the same. However, in contrast to the possible fine-tuning of the Higgs mass, it is difficult to imagine an anthropic reason for such a tuning in this context (notice that $\sigma$ cannot be the Higgs because the linear coupling is not compatible with gauge invariance).

Even if such a fine tuning occurs, a detectable signal requires a coincidence of $m \sim \mu \sim$ $H$, which itself calls for a physical explanation. Specifically, by measuring the trispectrum one could determine $\mu$ and $\Lambda$ independently [11,49] so that $\mu \sim H$ could be checked explicitly. While there are radiative corrections that would generate $m$ from $\mu$, these are suppressed by $\left(16 \pi^{2}\right)^{-1}$ and would themselves be insufficient to explain the coincidence of $\mu$ and $m$. Therefore, spoofing the signal with a finely-tuned, non-supersymmetric scalar requires that three physically unrelated quantities $-m, \mu$ and $H-$ are all accidentally of the same order of magnitude. This coincidence would be all the more surprising given that $H$ is a time-dependent quantity.

Some supersymmetric scenarios are even harder to spoof. For example, in the scenario with multiple light chiral multiplets, we expect many scalars to have $m \sim H$, not simply one. If more than one scalar couples to the inflaton, the measured bispectrum will differ from that of a single scalar. Without supersymmetry, the existence of many such light ${ }^{9}$ scalars seems highly implausible .

The signatures of models with many fields with $m \sim H$ will ultimately depend on the spectrum of masses and couplings. The dominant contribution to the equilateral configurations will arise from the fields with the largest couplings to $\phi$ (and to a lesser extent, size of $\mu$ ). On the other hand, the signal to noise in the squeezed limit will be power law suppressed for the more massive fields. The dominant contribution in both equilateral and squeezed configurations may therefore arise from different fields entirely. For some distributions of couplings, it is possible that equilateral configurations will be enhanced by the number of fields, potentially making the squeezed limit of the lightest field unmeasurable, but all such conclusions are model dependent.

The signature in the squeezed limit can also be mimicked by anomalous dimensions, as was shown in [12]. In this case, rather than a massive scalar coupled to $\phi$, we have some

\footnotetext{
${ }^{9}$ Massless scalars can be protected by a shift symmetry but produce the local shape $(\alpha=0)$.
} 

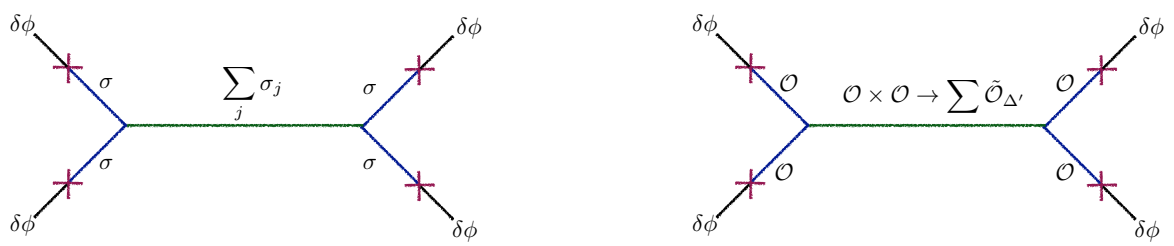

Figure 6. Schematic Feynman diagrams for the trispectrum of SUSY (left) and anomalous dimensions (right). The SUSY trispectrum gets contributions from the exchange of superpartners with $m_{i} \lesssim H$ where $\sigma$ is the linear combination that couples to $\phi$ and $\sigma_{i}$ are all superpartners that are coupled via cubic interactions with $\sigma$ (including $\sigma$ itself). When we have some operator $\mathcal{O}$, the trispectrum gets contributions from every operator $\tilde{\mathcal{O}}_{\Delta^{\prime}}$ that appears in the operator product expansion of $\mathcal{O}$ with itself. For interacting theories, these contributions should differ significantly from the weakly coupled scalars.

operator $\mathcal{O}$ with a scaling dimension $\Delta$. In the presence of $\mathcal{O}$ one finds the same behavior in the squeezed limit with $\alpha=\Delta$ when $\Delta \leq 2$.

In principle, the trispectrum offers the opportunity to distinguish these models. The trispectrum always gets contributions from exchanging the inflaton between two bispectra. However, when there are extra fields involved, there are additional contributions to the trispectrum from the exchange of these fields [49], as shown in figure 6. For SUSY, these contributions come from the exchange of $\sigma$ or any other superpartner. In this case, the trispectrum signal-to-noise is suppressed only by $\frac{\mu}{H}$ relative to the bispectrum and is potentially detectable. A similar phenomenon occurs when we couple to an operator $\mathcal{O}$, but now every operator in the operator product expansion contributes to the trispectrum [12]. These contributions are generically quite different from those of a weakly coupled scalar and could be used to distinguish between the two scenarios.

A final issue is the possibility that inflation took place in an excited state (i.e. not in the Bunch-Davies vacuum). As discussed in, e.g. [50-52], excited states lead to violations of the single field consistency condition and can produce measurable signals in the squeezed limit. However, achieving more than two orders of magnitude of scale-invariant perturbations is challenging due to back-reaction constraints $[53,54]$. The models can often be distinguished in the CMB which covers three orders of magnitude in scale.

As a whole, the prospects are closely analogous to low-energy probes such as EDMs and flavor violation. Although EDMs and flavor violation are promoted as suggestive indirect indications of supersymmetry, they are subject to similar ambiguities; a signal in low-energy channels could also be spoofed by physics unrelated to SUSY, such as an extended Higgs sector. Moreover, an EDM signal could even arise from non-supersymmetric degrees of freedom without implying additional fine-tuning, as in the case of new fermions generating EDMs from two-loop Barr-Zee diagrams [55]. In this respect an inflationary signal is perhaps more suggestive of supersymmetry than an EDM signal, since spoofing it with additional perturbative states requires further fine-tuning. But in both cases, the scales and interactions relevant for the signal are highly suggestive of supersymmetry and further measurements may help to disentangle various non-supersymmetric alternatives. 


\section{Outlook}

The observed Higgs mass and apparent success of supersymmetric gauge coupling unification suggests that supersymmetry is relevant during the inflationary era, even if it is broken well above the weak scale. In this work we have demonstrated the potential for next-generation measurements of cosmological observables to probe supersymmetry at high scales. A variety of generic and highly-motivated supersymmetric scenarios give rise to the signals of quasi-single field inflation - namely, equilateral non-gaussianity with a non-trivial squeezed limit bearing the imprint of an additional scalar with $m \sim H$ during inflation. The existence of such an additional scalar can be distinguished from single-field scenarios in next-generation measurements. Observation of a signal would be suggestive of supersymmetry at high scales, in close analogy with the prospects of low-energy probes such as EDMs and flavor violation.

The key observable in the search for SUSY is a bispectrum with an equilateral shape. This analysis is performed optimally in the CMB [40] but a similar analysis will be required in LSS surveys (which has not been performed to date). Galaxy surveys present a number of complications beyond the $\mathrm{CMB}$ and their ultimate reach remains to be determined, somewhat analogous to the challenges presented by the LHC compared to LEP. The amount of information in LSS vastly exceeds the CMB, provided we can address these issues.

Although the recent BICEP2 measurement of tensor modes provides strong motivation for probing supersymmetry during inflation, our analysis remains relevant even if the central value of $r$ changes substantially in future measurements. If the central value of $r$ is lowered, the scale $\Lambda$ probed by LSS and CMB polarization measurements changes according to (3.2). Our forecasting for $\alpha$ is unchanged. Should the signal of interest be observed - namely, modest non-gaussianity with evidence in the squeezed limit for a scalar of mass $m \sim H$ the interpretation in terms of split supersymmetry remains valid. Moreover, now an upper bound could be set on the scale of supersymmetry breaking in the current vacuum, much in the same way that observation of an anomalous EDM could place an upper bound on the scale of soft masses.

\section{Acknowledgments}

We are grateful to Nima Arkani-Hamed, Valentin Assassi, Daniel Baumann, and Liam McAllister for useful conversations. $\mathrm{NC}$ is supported by the DOE under grants DOESC0010008, DOE-ARRA-SC0003883, and DOE-DE-SC0007897. The research of D.G. is supported in part by the Stanford Institute for Theoretical Physics and by the U.S. Department of Energy contract to SLAC no. DE-AC02-76SF00515.

Open Access. This article is distributed under the terms of the Creative Commons Attribution License (CC-BY 4.0), which permits any use, distribution and reproduction in any medium, provided the original author(s) and source are credited. 


\section{References}

[1] BICEP2 collaboration, P.A.R. Ade et al., Detection of B-Mode Polarization at Degree Angular Scales by BICEP2, Phys. Rev. Lett. 112 (2014) 241101 [arXiv:1403.3985] [INSPIRE].

[2] S. Dimopoulos, S. Raby and F. Wilczek, Supersymmetry and the Scale of Unification, Phys. Rev. D 24 (1981) 1681 [INSPIRE].

[3] R. Kallosh and A.D. Linde, Landscape, the scale of SUSY breaking and inflation, JHEP 12 (2004) 004 [hep-th/0411011] [inSPIRE].

[4] A.H. Guth, The Inflationary Universe: A Possible Solution to the Horizon and Flatness Problems, Phys. Rev. D 23 (1981) 347 [InSPIRE].

[5] A.D. Linde, A New Inflationary Universe Scenario: A Possible Solution of the Horizon, Flatness, Homogeneity, Isotropy and Primordial Monopole Problems, Phys. Lett. B 108 (1982) 389 [INSPIRE].

[6] A. Albrecht and P.J. Steinhardt, Cosmology for Grand Unified Theories with Radiatively Induced Symmetry Breaking, Phys. Rev. Lett. 48 (1982) 1220 [INSPIRE].

[7] K.N. Abazajian et al., Inflation Physics from the Cosmic Microwave Background and Large Scale Structure, arXiv:1309.5381 [INSPIRE].

[8] J.M. Maldacena, Non-Gaussian features of primordial fluctuations in single field inflationary models, JHEP 05 (2003) 013 [astro-ph/0210603] [INSPIRE].

[9] P. Creminelli and M. Zaldarriaga, Single field consistency relation for the 3-point function, JCAP 10 (2004) 006 [astro-ph/0407059] [INSPIRE].

[10] X. Chen and Y. Wang, Quasi-Single Field Inflation and Non-Gaussianities, JCAP 04 (2010) 027 [arXiv:0911.3380] [INSPIRE].

[11] X. Chen and Y. Wang, Quasi-Single Field Inflation with Large Mass, JCAP 09 (2012) 021 [arXiv: 1205.0160] [INSPIRE].

[12] D. Green, M. Lewandowski, L. Senatore, E. Silverstein and M. Zaldarriaga, Anomalous Dimensions and Non-Gaussianity, JHEP 10 (2013) 171 [arXiv:1301.2630] [INSPIRE].

[13] D. Baumann and D. Green, Signatures of Supersymmetry from the Early Universe, Phys. Rev. D 85 (2012) 103520 [arXiv:1109.0292] [INSPIRE].

[14] J.D. Wells, PeV-scale supersymmetry, Phys. Rev. D 71 (2005) 015013 [hep-ph/0411041] [INSPIRE].

[15] N. Arkani-Hamed and S. Dimopoulos, Supersymmetric unification without low energy supersymmetry and signatures for fine-tuning at the LHC, JHEP 06 (2005) 073 [hep-th/0405159] [INSPIRE].

[16] G.F. Giudice and A. Romanino, Split supersymmetry, Nucl. Phys. B 699 (2004) 65 [Erratum ibid. B 706 (2005) 65-89] [hep-ph/0406088] [INSPIRE].

[17] N. Arkani-Hamed, Minimally Split SUSY, talk at SavasFest (2012), Bechtel Conference Center, Encina Hall, Stanford University, 18-19 May 2012, www.stanford.edu/dept/physics/events/2012/SavasFest/slides/Nima\%20Arkani-Hamed.pdf.

[18] A. Arvanitaki, N. Craig, S. Dimopoulos and G. Villadoro, Mini-Split, JHEP 02 (2013) 126 [arXiv:1210.0555] [INSPIRE]. 
[19] N. Arkani-Hamed, A. Gupta, D.E. Kaplan, N. Weiner and T. Zorawski, Simply Unnatural Supersymmetry, arXiv:1212.6971 [INSPIRE].

[20] V. Assassi, D. Baumann, D. Green and L. McAllister, Planck-Suppressed Operators, arXiv: 1304.5226 [INSPIRE].

[21] L. Iliesiu, D.J.E. Marsh, K. Moodley and S. Watson, Constraining SUSY with Heavy Scalars: using the CMB, Phys. Rev. D 89 (2014) 103513 [arXiv:1312.3636] [INSPIRE].

[22] Particle Data Group collaboration, J. Beringer et al., Review of Particle Physics (RPP), Phys. Rev. D 86 (2012) 010001 [INSPIRE].

[23] G.F. Giudice and A. Strumia, Probing High-Scale and Split Supersymmetry with Higgs Mass Measurements, Nucl. Phys. B 858 (2012) 63 [arXiv:1108.6077] [INSPIRE].

[24] C. Cheung, L.J. Hall, D. Pinner and J.T. Ruderman, Prospects and Blind Spots for Neutralino Dark Matter, JHEP 05 (2013) 100 [arXiv:1211.4873] [INSPIRE].

[25] T. Cohen, M. Lisanti, A. Pierce and T.R. Slatyer, Wino Dark Matter Under Siege, JCAP 10 (2013) 061 [arXiv: 1307.4082] [INSPIRE].

[26] J. Fan and M. Reece, In Wino Veritas? Indirect Searches Shed Light on Neutralino Dark Matter, JHEP 10 (2013) 124 [arXiv:1307.4400] [inSPIRE].

[27] P. Fox, A. Pierce and S.D. Thomas, Probing a QCD string axion with precision cosmological measurements, hep-th/0409059 [INSPIRE].

[28] ACME collaboration, J. Baron et al., Order of Magnitude Smaller Limit on the Electric Dipole Moment of the Electron, Science 343 (2014) 269 [arXiv:1310.7534] [INSPIRE].

[29] D. McKeen, M. Pospelov and A. Ritz, Electric dipole moment signatures of PeV-scale superpartners, Phys. Rev. D 87 (2013) 113002 [arXiv:1303.1172] [inSPIRE].

[30] W. Altmannshofer, R. Harnik and J. Zupan, Low Energy Probes of PeV Scale Sfermions, JHEP 11 (2013) 202 [arXiv:1308.3653] [INSPIRE].

[31] UTFIT collaboration, M. Bona et al., Model-independent constraints on $\Delta F=2$ operators and the scale of new physics, JHEP 03 (2008) 049 [arXiv:0707.0636] [INSPIRE].

[32] D. Baumann and D. Green, Supergravity for Effective Theories, JHEP 03 (2012) 001 [arXiv: 1109.0293] [INSPIRE].

[33] D.H. Lyth, What would we learn by detecting a gravitational wave signal in the cosmic microwave background anisotropy?, Phys. Rev. Lett. 78 (1997) 1861 [hep-ph/9606387] [INSPIRE].

[34] D. Baumann and D. Green, A Field Range Bound for General Single-Field Inflation, JCAP 05 (2012) 017 [arXiv: 1111.3040] [inSPIRE].

[35] C. Cheung, P. Creminelli, A.L. Fitzpatrick, J. Kaplan and L. Senatore, The Effective Field Theory of Inflation, JHEP 03 (2008) 014 [arXiv:0709.0293] [INSPIRE].

[36] L. Senatore and M. Zaldarriaga, The Effective Field Theory of Multifield Inflation, JHEP 04 (2012) 024 [arXiv: 1009.2093] [INSPIRE].

[37] E. Sefusatti, M. Liguori, A.P.S. Yadav, M.G. Jackson and E. Pajer, Constraining Running Non-Gaussianity, JCAP 12 (2009) 022 [arXiv: 0906.0232] [INSPIRE].

[38] J.J.M. Carrasco, S. Foreman, D. Green and L. Senatore, The Effective Field Theory of Large Scale Structures at Two Loops, arXiv:1310.0464 [INSPIRE]. 
[39] E. Sefusatti, J.R. Fergusson, X. Chen and E.P.S. Shellard, Effects and Detectability of Quasi-Single Field Inflation in the Large-Scale Structure and Cosmic Microwave Background, JCAP 08 (2012) 033 [arXiv: 1204.6318] [INSPIRE].

[40] Planck collaboration, P.A.R. Ade et al., Planck 2013 Results. XXIV. Constraints on primordial non-Gaussianity, arXiv:1303.5084 [INSPIRE].

[41] CMBPol Study Team collaboration, D. Baumann et al., CMBPol Mission Concept Study: Probing Inflation with CMB Polarization, AIP Conf. Proc. 1141 (2009) 10 [arXiv:0811.3919] [INSPIRE].

[42] J. Norena, L. Verde, G. Barenboim and C. Bosch, Prospects for constraining the shape of non-Gaussianity with the scale-dependent bias, JCAP 08 (2012) 019 [arXiv:1204.6324] [INSPIRE].

[43] R. Scoccimarro, E. Sefusatti and M. Zaldarriaga, Probing primordial non-Gaussianity with large-scale structure, Phys. Rev. D 69 (2004) 103513 [astro-ph/0312286] [INSPIRE].

[44] D. Babich, P. Creminelli and M. Zaldarriaga, The shape of non-Gaussianities, JCAP 08 (2004) 009 [astro-ph/0405356] [INSPIRE].

[45] EUCLID collaboration, R. Laureijs et al., Euclid Definition Study Report, arXiv:1110.3193 [INSPIRE].

[46] N. Dalal, O. Dore, D. Huterer and A. Shirokov, The imprints of primordial non-Gaussianities on large-scale structure: scale dependent bias and abundance of virialized objects, Phys. Rev. D 77 (2008) 123514 [arXiv:0710.4560] [INSPIRE].

[47] F. Schmidt and M. Kamionkowski, Halo Clustering with Non-Local Non-Gaussianity, Phys. Rev. D 82 (2010) 103002 [arXiv: 1008.0638] [INSPIRE].

[48] T. Baldauf, U. Seljak and L. Senatore, Primordial non-Gaussianity in the Bispectrum of the Halo Density Field, JCAP 04 (2011) 006 [arXiv:1011.1513] [INSPIRE].

[49] V. Assassi, D. Baumann and D. Green, On Soft Limits of Inflationary Correlation Functions, JCAP 11 (2012) 047 [arXiv: 1204.4207] [INSPIRE].

[50] R. Holman and A.J. Tolley, Enhanced Non-Gaussianity from Excited Initial States, JCAP 05 (2008) 001 [arXiv:0710.1302] [INSPIRE].

[51] J. Ganc, Calculating the local-type fNL for slow-roll inflation with a non-vacuum initial state, Phys. Rev. D 84 (2011) 063514 [arXiv:1104.0244] [INSPIRE].

[52] D. Chialva, Signatures of very high energy physics in the squeezed limit of the bispectrum (violation of Maldacena's condition), JCAP 10 (2012) 037 [arXiv:1108.4203] [INSPIRE].

[53] R. Flauger, D. Green and R.A. Porto, On squeezed limits in single-field inflation. Part I, JCAP 08 (2013) 032 [arXiv: 1303.1430] [INSPIRE].

[54] A. Aravind, D. Lorshbough and S. Paban, Non-Gaussianity from Excited Initial Inflationary States, JHEP 07 (2013) 076 [arXiv: 1303.1440] [INSPIRE].

[55] S.M. Barr and A. Zee, Electric Dipole Moment of the Electron and of the Neutron, Phys. Rev. Lett. 65 (1990) 21 [Erratum ibid. 65 (1990) 2920] [INSPIRE]. 